\title{
The lifestyle of our kids (LOOK) project: Outline of methods
}

Richard D. Telford a,b,c,*, Shona L. Bass ${ }^{e}$, Marc M. Budge ${ }^{c, d}$, Donald G. Byrne ${ }^{c}$, John S. Carlson ${ }^{m}$, David Coles ${ }^{d}$, Ross B. Cunningham ${ }^{c}$, Robin M. Daly ${ }^{e}$, David W. Dunstan ${ }^{e, j}$, Rowena English ${ }^{e, o}$, Robert Fitzgerald $^{\mathrm{g}}$, Prisca Eser ${ }^{\mathrm{e}}$, Karen J. Gravenmaker ${ }^{\mathrm{d}}$, Wayne Haynes ${ }^{k}$, Peter E. Hickman ${ }^{c, d}$, Ahmad Javaid ${ }^{d}$, Xiaoli Jiang ${ }^{i}$, Tony Lafferty c,d, Mark McGrath ${ }^{\mathrm{k}}$, Mary Kay Martin a,b, Geraldine A. Naughton', Julia M. Potter ${ }^{c, d}$, Stacey J. Potter ${ }^{d}$, Laurence Prosser $^{p}$, David B. Pyne ${ }^{f}$, Graham J. Reynolds ${ }^{c, d}$, Philo U. Saunders a,b,f, Markus J. Seibel ${ }^{n}$, Jonathan E. Shaw ${ }^{j}$, Emma Southcott ${ }^{d}$, Wichat Srikusalanukul ${ }^{d}$, Darryl Stuckeyg, Rohan M. Telford ${ }^{\text {a,b }}$, Kerry Thomas ${ }^{c}$, Ken Tallis ${ }^{\mathrm{h}}$, Paul Waring ${ }^{\mathrm{c}}$

\footnotetext{
a The Commonwealth Institute, Australia

b The Commonwealth Institute, UK

c Australian National University, Australia

d The Canberra Hospital, Australia

e Deakin University, Australia

f Australian Institute of Sport, Australia

s University of Canberra, Australia

$\mathrm{h}$ The Australian Institute of Health and Welfare, Australia

i University of Ballarat, Australia

$\mathrm{j}$ International Diabetes Institute, Australia

k The Bluearth Institute, Australia

' Australian Catholic University, Australia

m Victoria University, Australia

n University of Sydney, Australia

- La Trobe University, Australia

P KIDS Foundation, Australia
} 


\section{Background}

Longitudinal and intervention-based data on the effects of low physical activity on the health and development in children are sparse. Also lacking clarity in children is the effect of early lifestyle on subsequent health through to adolescence and adulthood. A recent extended commentary in reference to physical activity and health stated "Unfortunately few researchers are conducting physical activity intervention studies (with biomarker outcomes) that could yield new behavioural interventions" and "intervention studies are needed for definitive testing". 1

Trudeau and Shephard ${ }^{2}$ have highlighted the potential importance of school-based programs in developing life-long attitudes to physical activityrelated behaviour. They wrote "Future research should address factors influencing the change of perceptions as a child matures. In addition to offering a quality physical education programme, schools should ensure that the total weekly amount of physical education is sufficient not only to maintain but also to enhance a child's physical fitness. More research is needed to determine the ability of school physical education programmes to influence physical activity behaviour in adult life and to evaluate strategies that will make optimal use of the curricular time allocated to physical education'”.
The primary aim of the lifestyle of our kids (LOOK) project is to investigate relationships of (a) physical activity in general, and (b) an externally provided specialist physical education program in schools, with physiological and psychological health and development in young children. This study is intended to provide a range of integrated scientific evidence upon which conclusions may be drawn in regard to optimising childhood health and development.

In outlining this multi-institutional, multidisciplinary project incorporating both observational as well as intervention community-based research, we hope to stimulate constructive criticism, suggestions, advice and collaboration. Moreover, as several of our initial publications of baseline data will concern isolated sections of the LOOK project, citation of a methods overview is an efficient way to describe the overall design and hypotheses.

\section{The LOOK study hypotheses}

The LOOK study will test two hypotheses:

(a) The quantity and quality of physical activity over 4 years in a cohort of children initially aged $7-8$ years will influence psychological and physical health and development. 


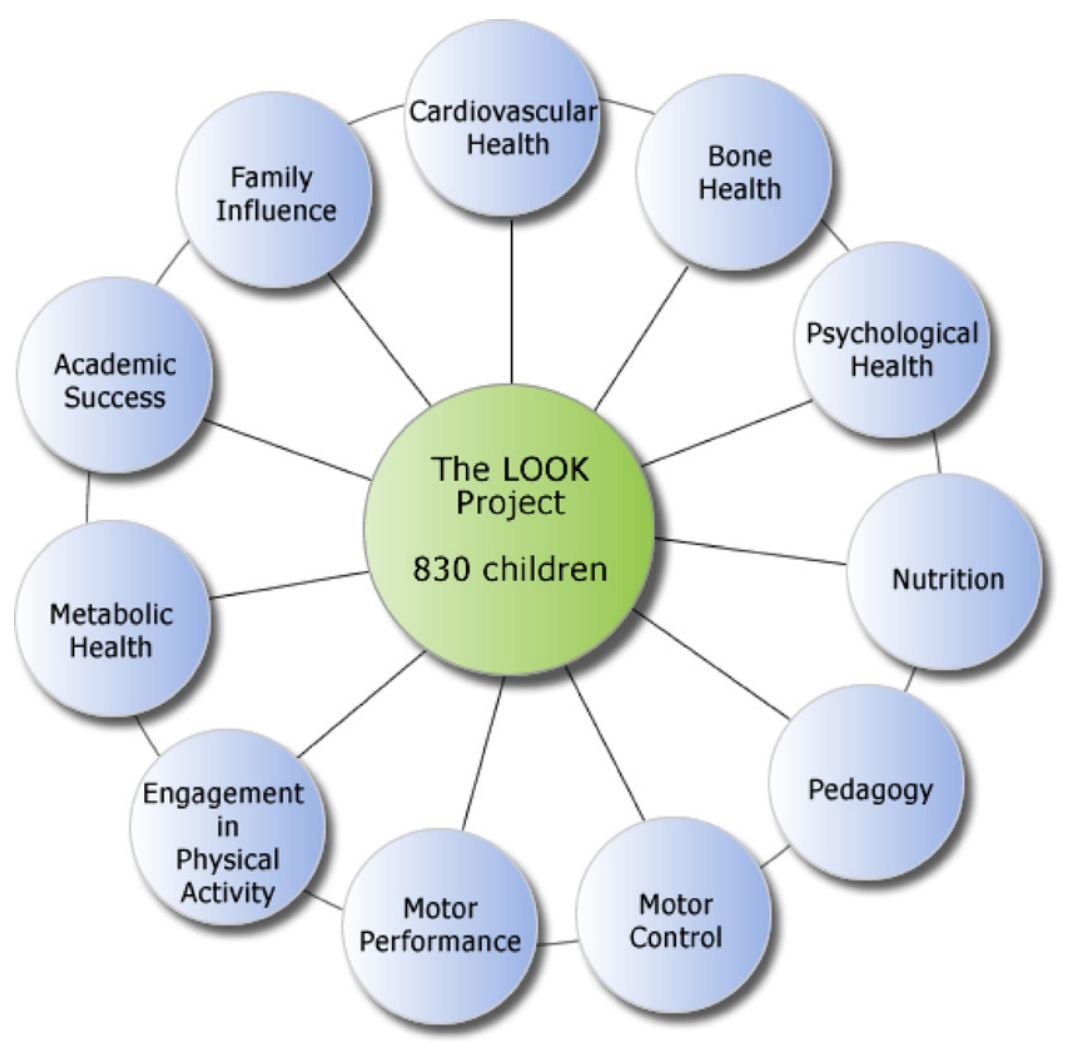

Figure 1 Components of the LOOK project.

(b) Compared with existing practices in primary schools, a physical education program enhanced by visiting specialist staff will improve psychological and physiological health and development, and lead to a more positive perception of, and participation in physical activity.

\section{Basic strategy, design and measurements}

Our investigations are outlined below and summarised in Fig. 1. While this longitudinal study, ending in 2009, with children at age 11-12 years, is complete in itself, the current project sets up an opportunity for ongoing monitoring into later life. Children $(n=830)$ aged $7-8$ years were recruited from outer suburb primary schools in Canberra, where the average household income approximates the mean for Australian city dwellers. In relation to hypothesis (a) we will examine longitudinal data to develop relationships between physical activity and the health and development variables from all 830 children. In relation to hypothesis (b) we randomly divided 30 schools into control $(n=400)$ and intervention groups $(n=430)$, the latter number of children being higher to account for the greater likelihood of school transfers affecting the intervention group. The intervention group receives the externally administered physical education program, as outlined below, two classes of $50 \mathrm{~min}$ being provided as part, not in addition to the "normal" weekly physical education set down by ACT Department of Education at 120 min per week. The control

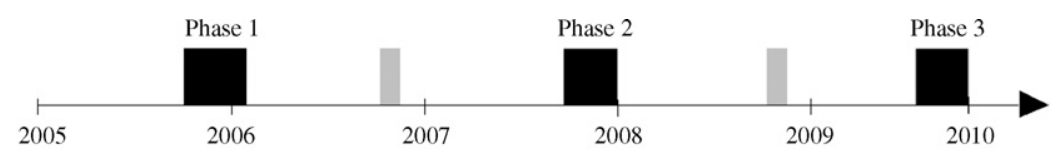

Testing period for all measures

Additional testing period for Physical Activity and Fitness

Figure 2 Time-line showing occurrence of various tests in LOOK. 
schools continue with their normal physical education, taken by the class-room teachers. The timing of major LOOK measurements is shown in Fig. 2.

Various aspects of this project has been approved by The Canberra Hospital, Australian Institute of Sport, Australian National University and Deakin University Ethics Committees and written informed consent was obtained from all parents, children and teachers being advised that they were able to withdraw from any test at any time. Provisions are in place, including coding of individual results, to ensure security and confidentiality of data.

\section{The measurements}

\section{Cardiovascular structure and function}

Endothelial dysfunction is an early event that predisposes individuals to formation of atherogenic plaques, and increased stiffness of the carotid artery and has already been found in children. Two sets of measures will be undertaken to characterise the vascular structure and function of the children using cardiac and carotid echo measures, as well as pulse wave analysis measures.

Echocardiography is performed using a Siemens Acuson Cypress ultrasound system with 7.0 and $3.5 \mathrm{MHz}$ transducers. Conventional M-mode, twodimensional and Doppler echocardiography techniques are employed to acquire digital images with children in a lateral decubitus position. Cardiac parameters are then measured off-line according to the American Society of Echocardiography guidelines. ${ }^{3,4}$ These include left ventricular dimensions and indices of left ventricular systolic and diastolic function. Carotid ultrasound is performed using the Siemens Acuson Cypress ultrasound system with a $7.0 \mathrm{MHz}$ vascular transducer. The maximum and mean far-wall intima media thickness is measured for the right and left common carotid arteries. Pulse wave analysis is employed to capture the radial and carotid pulse wave forms (SphygmoCor-Atcor Medical Systems, Sydney, Australia) and derive measures of aortic stiffness (e.g. augmentation index) and left ventricular function. ${ }^{5}$ The pulse waves are being recorded using a Millar applanation tonometer connected to the SphygmoCor apparatus and haemodynamic parameters are derived, together with peripheral brachial blood pressures using the system software, SphygmoCor Version 7.1. Our methods are modifications of those previously outlined. ${ }^{6}$

\section{Blood markers of degenerative diseases}

Indicators of risk of cardiovascular disease and type 2 diabetes are assessed from blood samples taken from a forearm vein in a standardised sitting posture, following overnight fasting. Phlebotomy is performed by experienced paediatric nurses. Standard blood markers being measured include plasma glucose, glycated haemoglobin and serum concentrations of HDL and LDL cholesterol, triglycerides, and insulin. We are also measuring plasma concentrations of C-reactive protein, homocysteine, and uric acid. In addition, blood is being stored for potential measurement of chemistry associated with inflammation, metabolic dysfunction, oxidative stress and we hope to gain funding for genetic studies. Delaying these assays to the end of the study has two advantages. First it allows samples from the various years to be assayed together, thereby eliminating any between-assay variation. Secondly, we are likely to make more informed decisions as to the most relevant studies to perform in healthy young children within the limits of available funding. All measures are performed in the Pathology Department Laboratories at The Canberra Hospital.

\section{Psychological influences on lifestyle and health}

Our investigations include (a) the influence of psychological factors on children's engagement in physical activity and (b) the effect of both level of physical activity and the intervention on mood, self-esteem, body image, and health risk attitudes and behaviours. Instruments include The Children's Stress Questionnaire, The Children's Body Image Scale, The Positive and Negative Affect Schedule (all modified for children by co-author D.G. Byrne), The Children's Depression Inventory, ${ }^{7}$ and The Self-Description Questionnaire (SDQ) for selfesteem-short version. ${ }^{8}$ Two additional tests will be applied in the second round of testing, a further assessment of self-esteem (using the SDQ1), and an assessment of attitudes to and perceived salience of health facilitating behaviours using a children's modification of The Perkins Adolescent Risk Screen. ${ }^{9}$

\section{Motor control}

Characterisation of perceptual style is carried out using a computerized version of the rod and frame test, where children, through a viewing tube, attempt to align a rod surrounded by a tilted frame to the vertical position. Children who closely esti- 
mate vertical are termed field-independent and those who cannot are termed field-dependent). ${ }^{10}$ Balance control is determined by children holding five different postures on a large rocker board, of similar shape and size to a surfboard. Board deflection, indicating balance, is detected by a fine wire sensor and is stored in a laptop computer. Fine motor control is assessed using the nut and bolt test, ${ }^{11}$ handedness and eye dominance noted during this procedure. Hand-eye coordination was measured with an indoor throw-rebound catch procedure developed for this study.

\section{Anthropometry and body composition}

Total body and regional body composition is measured using dual energy X-ray absorptiometry (DXA, Hologic Discovery QDR Series, Hologic Inc., Bedford, MA, USA). Total body scans are analysed using Hologic QDR System Software Version 12.4. DXA provides data on three body compartments; bone (mineral content and density), lean tissue mass and fat mass. ${ }^{12}$ Height is measured by a portable stadiometer using standard techniques (head in Frankfort plane) to the nearest $0.001 \mathrm{~m}$ and body mass is measured with portable electronic scales to the nearest $0.05 \mathrm{~kg}$.

\section{Components of bone strength}

Total body and regional areal bone mineral density (BMD) are measured using DXA, as described above. Bone geometry and volumetric $B M D$ are also assessed at the tibia and radius using peripheral quantitative computed tomography (PQCT), Stratec XCT 3000 scanner (Stratec Medical, Pforzheim, Germany). In contrast to DXA, PQCT allows separate assessment of trabecular and cortical bone compartments with regard to bone size, geometry, and volumetric BMD. From these data, measures of compression, bending and torsional strength of long bones will be calculated and limb cross-sectional areas of muscle and fat determined.

\section{Fitness assessment}

The results of any fitness testing have to be interpreted with caution, not only because fitness can be defined and measured in so many different ways, but also because maximal effort tests depend partly on motivation. This is especially true in children whose fitness test performances are more likely to be influenced by external factors, ${ }^{13}$ including their peers. We have endeavoured to minimise potential confounders from one assessment period to another by providing consistent motivational cues, and con- ducting tests in similar conditions. The $20 \mathrm{~m}$ shuttle run is used to estimate the aerobic fitness, single effort lower body power is measured by a vertical jump, and trunk (core) strength is measured using the duration the child can hold a self-supported prone (plank) position on forearms and toes.

\section{Measurement of physical activity}

Measuring physical activity is a challenging task, particularly in a large group of young children. To assist in our estimation of physical activity, all children undergo 7-day pedometer assessments. To estimate vigorous physical activity, questionnaires are administered to the parents to gauge the extent of family and community-based sporting and active recreation pursuits. Video analysis and observation of individual children are used to provide additional information in the physical education lessons in all schools. AT pedometers (New-Lifestyles, Lee's Summit, Missouri, USA) are used to assess physical activity, this pedometer type deemed sufficiently valid and reliable ${ }^{14}$ and recommended for use with children. Those unable to provide at least 3 days of pedometer data are retested, wearing the pedometer for an additional 7 days.

\section{Family involvement and medical history}

A questionnaire for parents was devised to gather information on child and family medical history, ethnicity, weight and height at birth, feeding history, health of mother during pregnancy. We also included questions on parents' involvement and attitudes toward their own and their children's sport and active leisure pursuits, the amount of time spent with television and video games, family meal patterns, beverage consumption and sleep patterns of the child.

\section{Nutritional intake}

Previously reported methodology ${ }^{15}$ was modified, and a 24 -h record used to measure food and nutrient intake. Detailed instructions are provided to parents and teachers on how to measure the food and beverages using provided household measuring cups and spoons. Foods consumed were coded for analysis using Foodworks Professional nutrition analysis software system (Xyris Software Pty Ltd., Qld) and energy intake, macronutrients and micronutrients calculated for each child. A review of the 24$\mathrm{h}$ dietary record procedure used in children and its strengths and limitations has been described elsewhere. ${ }^{16}$ 


\section{Other measurements}

\section{Academic achievement}

There is some concern that time spent with physical education in primary school may impede academic progression. We will investigate the legitimacy of this concern, at least in relation to literacy and numeracy, using the nationally compulsory assessment of numeracy and literacy administered to all children in grades 3,5 , and 7 .

\section{Pubertal assessment and skeletal age}

Physical maturation will be self-assessed using standard forms previously reported, ${ }^{17}$ using the five-stage standard developed by Tanner. ${ }^{18}$ Skeletal age will be assessed using the standard wrist X-ray technique.

\section{The movement education program and pedagogy}

The intervention is an externally administered physical activity program called Bluearth Discovery (Bluearth Institute, 1a Bowen Crescent, Melbourne, Vic., http://www.bluearth.org). The program incorporates components relevant to modern physical education, having been designed by physical education and sports science personnel from tertiary education and practical settings, emphasising maximal engagement and enjoyment for all children, irrespective of skill or fitness. Our pedagogy research group will investigate the influence of teacher styles and attitudes toward physical activity on the health and fitness and health outcomes of the children, adopting a mixed methods approach including surveys, case studies, teacher and pupil interviews and class observations. Teacher interviews in both intervention and control schools are used to gather information on their attitudes and self-efficacy in relation to their teaching of physical education. ${ }^{19}$ Part of the externally driven Discovery program is training the class-room teachers to teach Discovery classes themselves as the year progresses and the effectiveness of this professional development will be also assessed. Direct observation of physical education classes takes place using the "System of Observing Fitness Instruction Time"' (SOFIT) tool. ${ }^{20}$

\section{Data analyses}

Our longitudinal design allows direct estimation of relationships between changing physical activ- ity levels and changes in the health outcomes. This presents an advantage over cross-sectional studies where relationships we may be obscured by the variability between children. A variety of statistical models will be employed appropriate to each sub-study including (a) classic and generalised linear modelling, (b) general linear mixed modelling to account for structure in the data arising from the multilevel design, and (c) time-dependent modelling exploiting the longitudinal nature of the data as well as accounting for possible serial dependencies arising from repeat measures on the same subjects. Limitations in validity and reliability of testing are among the reasons studies such as ours require a relatively large number of children. Because the population prevalence and the strengths of effects will differ from module to module, it is not meaningful to perform a single, indicative power calculation in relation to hypothesis (a) or (b) above. Experience gathered from international studies suggests that our starting sample numbers of 830 , allowing for inevitable attrition, will support the rigorous application of statistical modelling required to test the two hypotheses described above. Our initial sample represented approximately $27 \%$ of all grade 2 children enrolled in ACT Government schools.

\section{Challenges}

Any large longitudinal study has many challenges, communication and subject recruitment/retention being two that stand out. Communication with parents, teachers and school principals is not straightforward as electronic mail may not be available nor read regularly. Letters of consent and special requirements for testing, sent home with the children, may be lost or ignored so perseverance with communication, patience and allowance for delays is required. Recruiting and retention of subjects has also demanded considerable attention. Our venous blood sampling is a potential deterrent to the children and together with bone and heart scans, the safety and importance of these procedures must be carefully explained to parents in easily understood terms. It is here that gaining the interest and cooperation of the school principal and class-room teacher is very important, their influence having a direct bearing on recruitment and retention success. Any form of embarrassment or stress for the child clearly jeopardises retention. Consequently we have played down inter-individual comparison of test results, emphasising personal improvement. 
Maximising retention will always be a greater challenge in control, rather than intervention schools, so our strategy has been to focus publicity and communication on the observational study rather than the intervention. The other factor we hope assists retention is the regular provision of easily understood test reports, including confidential advice to parents of any suspected medical abnormalities.

\section{Summary}

This methods paper summarises the Commonwealth Institute LOOK study, a 4-year, multidisciplinary longitudinal investigation of the relationships between physical activity and the health, psychological and physical development of a cohort of 830 children with a starting age of 7-8 years. This project also investigates a physical activity program provided to schools by specialist visiting physical educators in conjunction with the class-room teachers, comparing its effect with the normal practice of physical education undertaken by the class-room teachers.

\section{Acknowledgements}

We appreciate the advice provided by The Commonwealth Institute Advisory Board comprising Dr. Peter Frost (Commonwealth Institute), Professor Donald McIntyre (Cambridge University, UK), Professor Timothy Noakes (Cape Town University, South Africa), Associate Professor Heather Mackay (University of Vancouver, Vancouver, Canada), Mr. Mark Peters (Australian Sports Commission, Bruce, ACT, Australia), Professor Steven Blair (University of South Carolina, USA), and Mr. Malcolm Freake (Bluearth Institute, Melbourne, Vic., Australia). We also acknowledge the advice from Professor Terry Dwyer (Murdoch Institute), Professor Roland Stocker (University of NSW), Professor David Calermajer (University of Sydney), and Dr. J. Simons (Bluearth Institute).

We gratefully acknowledge the encouragement and funding support from the Board of Trustees of the Commonwealth Institute (UK), the Board of the Commonwealth Institute (Australia), the Australian Research Council, administrative support from the ACT Department of Education, and the provision of the coaches and delivery of the Discovery program by the Bluearth Institute.

\section{References}

1. Prentice RL, Willett WC, Greenwald P, Alberts D, Bernstein L, Boyd NF, et al. Nutrition and physical activity and chronic disease prevention: research strategies and recommendations. J Natl Cancer Inst 2004;96(17):1276-87.

2. Trudeau F, Shephard RJ. Contribution of school programmes to physical activity levels and attitudes in children and adults. Sports Med 2005;35(2):89-105.

3. Gottdiener JS, Bednarz J, Devereux R, Gardin J, Klein A, Manning WJ, et al. American Society of Echocardiography recommendations for use of echocardiography in clinical trials. J Am Soc Echocardiogr 2004;17(10):1086-119.

4. Lang RM, Bierig M, Devereux RB, Flachskampf FA, Foster E, Pellikka PA, et al. Recommendations for chamber quantification: a report from the American Society of Echocardiography's Guidelines and Standards Committee and the Chamber Quantification Writing Group, developed in conjunction with the European Association of Echocardiography, a branch of the European Society of Cardiology. J Am Soc Echocardiogr 2005;18(12):1440-63.

5. McEniery CM, Yasmin, Hall IR, Qasem A, Wilkinson IB, Cockcroft JR. Normal vascular aging: differential effects on wave reflection and aortic pulse wave velocity: the Anglo-Cardiff Collaborative Trial (ACCT). J Am Coll Cardiol 2005;46(9):1753-60.

6. Millasseau SC, Kelly RP, Ritter JM, Chowienczyk PJ. Determination of age-related increases in large artery stiffness by digital pulse contour analysis. Clin Sci (London) 2002;103(4):371-7.

7. Kovacs M. The children's depression inventory. New York: Multi-Health Systems; 1992. p. vii-101.

8. Marsh HW, Ellis LA, Parada RH, Richards G, Heubeck BG. A short version of the Self Description Questionnaire II: operationalizing criteria for short-form evaluation with new applications of confirmatory factor analyses. Psychol Assess 2005; 17(1):81-102.

9. Adams CD, Perkins KC, Lumley V, Hughes C, Burns JJ, Omar HA. Validation of the Perkins Adolescent Risk Screen (PARS). $J$ Adolesc Health 2003;33(6):462-70.

10. Isableu B, Ohlmann T, Cremieux J, Amblard B. Differential approach to strategies of segmental stabilisation in postural control. Exp Brain Res 2003;150(2):208-21.

11. Van Waelvelde H, De Weerdt W, De Cock P, Smits-Engelsman BC. Aspects of the validity of the movement assessment battery for children. Hum Movement Sci 2004;23(1): 49-60.

12. Lu PW, Briody JN, Ogle GD, Morley K, Humphries IR, Allen $J$, et al. Bone mineral density of total body, spine, and femoral neck in children and young adults: a cross-sectional and longitudinal study. J Bone Miner Res 1994;9(9): 1451-8.

13. Naughton GA, Carlson JS, Greene DA. A challenge to fitness testing in primary schools. J Sci Med Sport 2006;9(1/2):40-5.

14. Beets MW, Patton MM, Edwards S. The accuracy of pedometer steps and time during walking in children. Med Sci Sports Exerc 2005;37(3):513-20.

15. Hands B, Parker H, Glasson C, Brinkman S, Read H. Physical activity and nutrition levels in Western Australian children and adolescents. Western Australian Government; 2004. p. $26-30$.

16. Livingstone MB, Robson PJ. Measurement of dietary intake in children. Proc Nutr Soc 2000;59(2):279-93.

17. Duke PM, Litt IF, Gross RT. Adolescents' self-assessment of sexual maturation. Pediatrics 1980;66(6):918-20. 
18. Tanner JM. Growth at adolescence. Oxford: Blackwell Scientific Publications; 1962. p. 140.

19. Morgan P, Bourke S, Thompson K. Physical educator's perceptions about physical education: an analysis of the prospective and practicing teacher. In: The annual con- ference of the Australian association for research in education. 2002.

20. McKenzie TL, Feldman H, Woods SE, Romero KA, Dahlstrom $V$, Stone EJ, et al. Children's activity levels and lesson context during third-grade physical education. Res $Q$ Exerc Sport 1995;66(3):184-93. 